\title{
Automatic Moving Object Segmentation with Accurate Boundaries
}

\author{
Jia Wang, Haifeng Wang, Qingshan Liu, and Hanqing Lu \\ National Laboratory of Pattern Recognition, Institute of Automation, \\ Chinese Academy of Sciences, Beijing 100080, China \\ \{wangjia, hfwang, qsliu, luhq\}@nlpr.ia.ac.cn
}

\begin{abstract}
This paper presents a layer-model based method to segment moving objects from image sequence with accurate boundaries. The segmentation framework involves three stages: Motion seed detection, Motion layer expansion and Motion boundary refinement. In the first stage, motion seeds, which determine the amount and initial position of motion layers, are detected by corner matching between consecutive frames, and classified by global motion analysis. In the second stage, the detected motion seeds are expanded into motion layers. To preserve the spatial continuity, an energy function is defined to evaluate the spatial smoothness and accuracy of the layers. Then, Graph Cuts technique is used to solve the energy minimization problem and extract motion layers. In the last stage, the extracted layers are combined with edge information to find accurate boundaries of moving objects. The proposed method is tested on several image sequences and the experimental results illustrate its promising performance.
\end{abstract}

\section{Introduction}

Moving object segmentation is very important for many video processing applications, such as video representation, analysis, compression and synthesis. In the past, a number of algorithms have been proposed, each of which has its particular features and applications.

Arch and Kaup [1] proposed a segmentation technique using a statistical approach. They model the difference of pixels in background as a gaussian distribution and change detection mask (CDM) is yielded by finding the frame difference. Since this technique relies on the intensity information, there are always hollows within the detected motion regions when there is no plenty of textures. This shortcoming is partly overcome by Mech and Wollborn in [2] using morphological closing operation.

Meier and Ngan proposed an automatic segmentation technique for moving objects using a binary image model [3]. The binary model is derived from an edge image and is updated every frame to keep the changes in location and shape. The detection of a moving object is based on the binary model matching between two consecutive frames using Hausdoff distance. The advantage of this technique lies in its capable of tracking an object that stops moving for a certain 
period of time. However, the segmentation results depend on the success of the initial segmentation at the first frame.

In [4], Nicolescu and Medioni have employed a tensor voting procedure to obtain piecewise smooth motion region. They used two successive frames as input. For every pixel in the first frame, a normalized cross- correlation procedure is used to produce candidate matches for the second image. Then, 4-D tensor voting is performed to find the best match motion vector. After that, another 2-D tensor voting is used to obtain the motion boundary. While in some cases this method gives fairly nice results, its accuracy is influenced by the initial computation of candidate matches. Furthermore, it has the same problem as [1] that the spatial continuity of motion regions also depends on the abundance of textures.

In this paper, a new algorithm is proposed for automatic moving object segmentation, which can solve the above problems. The segmentation scheme consists of three steps: Motion seed detection, Motion layer expansion and Motion boundary refinement. In the first stage, motion seeds, which determine the amount and initial position of motion layers, are detected by corner matching between consecutive frames, and classified by global motion analysis. In the second stage, the detected motion seeds are expanded into motion layers. During the expansion process, an energy function is defined to evaluate the spatial smoothness and accuracy of the motion layers, by means of what the layer can keep its spatial continuity even when there is no plenty of textures. Then, Graph Cuts technique is used to settle the energy minimization problem and expand the motion layers. In the last stage, the extracted layers are combined with edge information to find the accurate boundaries of moving objects.

\section{Motion Seed Detection}

Layered models [5][6][7][8] provide a natural way to detect motion areas with different velocities. Computationally, the problem is addressed by first estimating motion vectors for all the pixels, then pixels are grouped into different layers based on their motion cues. Thus, to extract motion layers, it is necessary to first find out how many motions are there in the video and where they are. In this paper, such motions are regarded as seeds for the further extracted motion layers.

In this section, we extract the motion seeds by tracking corners between consecutive frames.

First, Harris detector is performed to detect corners in the current frame. The detected corners are tracked back to the previous frame to find their correspondences. Based on the coordinate difference between corresponding corners, the motion vectors between them are achieved. Then, the extracted corners together with their motion vectors are regarded as motion seeds.

Since the extracted motion seeds are disordered, to distinguish those belonging to moving objects, global motion analysis is used to cluster them into global motion seeds and local motion seedsbased on their motion vectors, where global 
motion seeds have motion vectors consistent with global motion caused by the motion of camera, and local motion seeds have motion vectors corresponding to local motions caused by the moving objects.

For global motion analysis, a 3-parameter model is proposed to describe global motion, which can be expressed as

$$
\left[\begin{array}{l}
v_{x} \\
v_{y}
\end{array}\right]=a_{1}\left[\begin{array}{l}
x \\
y
\end{array}\right]+\left[\begin{array}{l}
a_{2} \\
a_{3}
\end{array}\right]
$$

In the above model, $(x, y)$ is a pixel's coordinates with respect to the center of the image, and $\left(v_{x}, v_{y}\right)$ is the motion vector of the pixel. The three parameters are

$$
a_{1}=z_{x y}, a_{2}=f_{1}\left(p_{x}, z_{x y}\right), a_{3}=f_{2}\left(p_{y}, z_{x y}\right)
$$

where $z_{x y}$ is the zoom factor of the moving camera and $\left(p_{x}, p_{y}\right)$ is the pan vector.

Based on the 3-parameter model, all the candidate motion seeds are compared with the global motion to find out whether they belong to the global motion or not. Such process is performed as follows:

STEP 1. The 3 parameters in formula (1) are estimated based on potential global motion seeds. (Note that for the first iteration, all the seeds are regarded as potential global motion seeds.)

Suppose there are $N$ potential global motion seeds. Let $\left(v_{x}^{k}, v_{y}^{k}\right)$ be the motion vector of a seed $k(k=0,1, \cdots, N-1)$, whose coordinate is $\left(s_{x}^{k}, s_{y}^{k}\right)$ with respect to the center of the frame. The parameters $\left(a_{1}, a_{2}, a_{3}\right)$ are estimated using the following criteria:

$$
\left(a_{1}, a_{2}, a_{3}\right)=\arg \min \sum_{k=0}^{N-1}\left[\left(v_{x}^{k}-a_{1} s_{x}^{k}-a_{2}\right)^{2}+\left(v_{y}^{k}-a_{1} s_{y}^{k}-a_{3}\right)^{2}\right]
$$

Differentiating (3) with respect to the parameters and setting the derivatives to zero, the following solution can be achieved as:

$$
\begin{gathered}
a_{1}=\frac{N \Psi_{1}-\Psi_{2}}{N \Psi_{3}-\Psi_{4}} \\
a_{2}=\Psi_{3} \sum v_{x}^{k}-\Psi_{1} \sum s_{x}^{k}+\frac{\Psi_{5}}{N} \sum s_{y}^{k} \\
a_{3}=\Psi_{3} \sum v_{y}^{k}-\Psi_{1} \sum s_{y}^{k}-\frac{\Psi_{5}}{N} \sum s_{x}^{k}
\end{gathered}
$$

where

$$
\begin{aligned}
& \Psi_{1}=\sum v_{x}^{k} s_{x}^{k}+\sum v_{y}^{k} s_{y}^{k}, \Psi_{2}=\sum v_{x}^{k} \sum s_{x}^{k}+\sum v_{y}^{k} \sum s_{y}^{k}, \\
& \Psi_{3}=\sum\left(s_{x}^{k}\right)^{2}+\sum\left(s_{y}^{k}\right)^{2}, \Psi_{4}=\left(\sum s_{x}^{k}\right)^{2}+\left(\sum s_{y}^{k}\right)^{2}, \\
& \Psi_{5}=\sum v_{y}^{k} \sum s_{x}^{k}+\sum v_{x}^{k} \sum s_{y}^{k} .
\end{aligned}
$$

STEP 2. Based on the estimated parameters $\left(a_{1}, a_{2}, a_{3}\right)$ by STEP 1, each potential global motion seed $k$ is checked by

$$
\Delta_{k}=\left(v_{x}^{k}-a_{1} s_{x}^{k}-a_{2}\right)^{2}+\left(v_{y}^{k}-a_{1} s_{y}^{k}-a_{3}\right)^{2}
$$


If $\Delta_{k}$ lies within a predefined threshold, seed $k$ will be maintained as a potential global motion seed. Otherwise, it will be regarded as a local motion seed.

STEP 3. Return to STEP 1 and re-estimate $\left(a_{1}, a_{2}, a_{3}\right)$ based on the remaining potential global motion seeds.

The detected motion seeds will be used as the initial state of motion layer expansion in the following section, where the local motion seeds will be expanded into motion layers corresponding to moving objects and global motion seeds to layers corresponding to background. Fig. 1 shows an example of detected motion seeds on Hall-monitor.

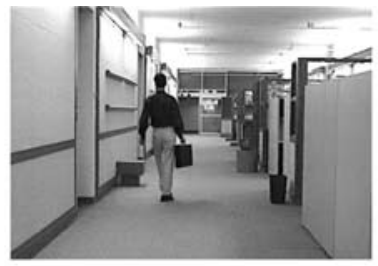

(a)

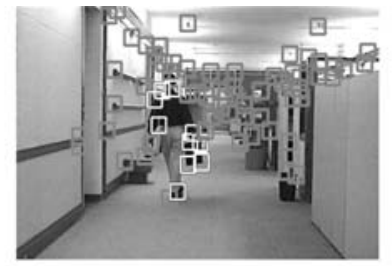

(b)

Fig. 1. Motion seed detection: the white squares illustrate the detected global motion seeds, and the black squares are the local motion seeds

\section{Motion Layer Expansion}

In this section, Graph cuts technique [9][10] is used to expand the motion layers from the detected motion seeds. Based on Graph cuts theory, motion layer expansion is regarded as an image-labelling process, where the labels correspond to motion vectors. During the labelling process, pixels belonging to different motion layers will have different labels.

\subsection{Energy Function Definition}

According to [8], many layer models have a weakness that each pixel is assigned to a layer independently of its neighbor pixels. As the result, the extracted layers always don't manifest the constraint that most physical objects are spatially coherent. In this paper, this spatial coherency constraint is formulated into an energy minimization problem, and then settled by graph cuts technique.

For the image-labelling process, the assigned labels should be consistent with the image data and be piecewise smooth, viz. they should remain unchanged or vary smoothly on the surface of an object, but change dramatically at object boundaries. Such problem can be described as: Finding a labelling $f$ that assigns each pixel $p \in P$ a label $f_{p} \in L$, where $f$ is both piecewise smooth and consistent with the observed data.In this paper, the above problem is formulated into an energy minimization problem with the energy function 


$$
E(f)=E_{\text {data }}(f)+E_{\text {smooth }}(f)
$$

Here $E_{\text {smooth }}$ evaluates the extent of how $f$ is piecewise smooth, while $E_{\text {data }}$ evaluates the disagreement between $f$ and the observed data. The form of $E_{d a t a}$ is typically

$$
\begin{gathered}
E_{\text {data }}(f)=\sum_{p \in P} D_{p}\left(f_{p}\right) \\
D_{p}\left(f_{p}\right)=\left|I_{\text {current }}(p)-I_{\text {previous }}(q)\right|
\end{gathered}
$$

where $I_{\text {current }}(p)$ is the intensity of $p$ in the current frame. And $q$ is the corresponding pixel of $p$ in the next frame. The $E_{\text {smooth }}$ is defined as

$$
\begin{gathered}
E_{\text {smooth }}(f)=\sum_{\{p, q\} \in P} V_{p, q}\left(f_{p}, f_{q}\right) \\
0 \text { if } f_{p}=f_{q} \\
V_{p, q}\left(f_{p}, f_{q}\right)=\left\{\begin{array}{c}
\text { const if } f_{p} \neq f_{q}
\end{array}\right.
\end{gathered}
$$

where $N$ is the set of interacting pairs of pixels. const is the energy evaluating the smoothness of adjacent labels.

\subsection{Motion Layer Expansion}

Graph cuts technique [9] is used to minimize the energy defined in (8). The construction of the graph is the same as that used in [10]. This section illustrates how to achieve motion layers from motion seeds using such method.

First, some of the graph nodes are labelled initially according to the different type of the motion seeds. All nodes corresponding to the global motion seeds will be assigned a uniform label $l_{0}$. Nodes corresponding to local motion seeds will be labelled independently: For to a local motion seeds $k$, with coordinates $\left(s_{x}^{k}, s_{y}^{k}\right)$ and motion vector $\left(v_{x}^{k}, v_{y}^{k}\right)$, its graph node will be assigned an special label $l_{k}$. Based on the labelled graph, the nodes assigned $l_{0}$ will be regarded as belonging to background, and nodes assigned other labels will be regarded as belonging to moving objects, whose motion is described by $\left(v_{x}^{k}, v_{y}^{k}\right)$. Then, the process of motion layer expansion from motion seeds can be summarized as:

STEP 1. Start with initial labelling $f$, where only the nodes corresponding to motion seeds are labelled;

STEP 2. Compute the $E(f)$ of (8);

STEP 3. Set success $:=0$;

STEP 4. For each label $l \in L$ :

4.1. Find $\hat{f}=\arg \min E\left(f^{c}\right)$ using Graph cuts technique;

4.2. If $E(\hat{f})<E(f)$, set $f:=\hat{f}$ and success $:=1$;

STEP 5. If success $=1$ goto STEP 2; else, goto STEP 6;

STEP 6. Output the final $f$.

In the final $f$, each node in the graph is assigned a label corresponding to its motion, based on what motion layers are naturally extracted. Pixels who were assigned label $l_{0}$ are segmented as background. Then, the rest pixels with other labels can be regarded as the moving objects. The motion layers extracted for Fig. 1 are shown in Fig. 2. it can be seen that the spatial continuity of layers is well preserved by graph cuts technique. According to Fig. 2, it is clear that 


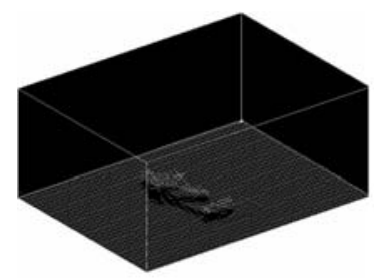

(a)

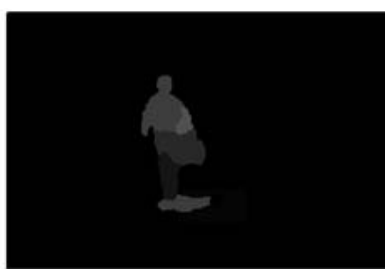

(b)

Fig. 2. Motion layers: (a) shows the extracted motion layers in 3-D space, where the horizontal dimensions correspond to $(x, y)$ coordinates in image plane and vertical dimension $v$ correspond to $v=\left(v_{x}^{2}+v_{y}^{2}\right)^{1 / 2}$. The extracted layers can also be described by velocity map as shown in (b), where a higher velocity $v$ has a higher intensity.

\section{Motion Boundary Refinement}

When the motion layers are extracted, the objects can be roughly identified by their motion, but the extracted motion layers may still be inaccurate along the motion boundaries. This section will combine edge information to refine the motion boundaries.

In this paper, morphological watershed transform [11] is used to detect intensity edges, which can produce an image partition with regions enclosed by onepixel-wide contours. To deal with the over-segmentation problem of watershed segmentation, a region merging method presented in [12] is used to post-process the image.

Suppose the current image is partitioned into $n$ regions $\Re=\left\{R_{1}, R_{2}, \cdots, R_{n}\right\}$, and every region $R_{i}$ is enclosed by a one-pixel-wide edge $\overline{E_{i}}$. Based on the segmented edge map, following information are calculated: $\left\|R_{i}\right\|$, area of region $R_{i} ;\left\|\overline{E_{i}}\right\|$, length of edge $\overline{E_{i}}$. On the other hand, velocity map provides us an initial Object Mask (OM), regions not labeled by $l_{0}$, with rough boundaries. Fig. 3(a) shows the initial OM by white regions. Then, considering the edge map together with the initial OM, object segmentation is regarded as a region classification process, in which all the regions will be classified into two groups: object regions and background regions. The classification is based on

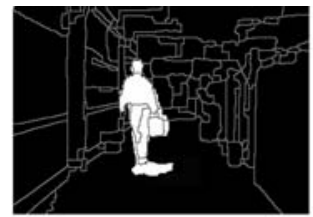

(a)

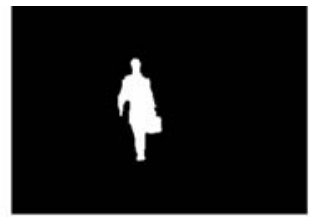

(b)

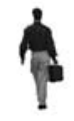

(c)

Fig. 3. Moving object extraction: (a) shows the initial OM and the edge map; (b) is the refined OM; the extracted object is shown in (c) 
the motion analysis from both the regions and the edges. Two criteria are defined: $R P_{i}=\left\|R_{i}^{*}\right\| /\left\|R_{i}\right\|$, where $R_{i}^{*}=\left\{p \mid p \in O M, p \in R_{i}\right\} ; E P_{i}=\left\|\overline{E_{i}^{*}}\right\| /\left\|\overline{E_{i}}\right\|$, where $\overline{E_{i}^{*}}=\left\{p \mid p \in O M, p \in \overline{E_{i}}\right\}$. Considering the possible inaccuracy of initial $\mathrm{OM}$ at boundaries, regions are classified as:

$A$ region $R_{i}$ is determined to be a object region, if, and only if, $R P_{i}>$ threshold $_{R} \& E P_{i}>$ threshold $_{E}$.

In our experiments, the thresholds are simply selected as threshold ${ }_{R}=$ threshold $E=0.7$. Based on the above criterion, all the regions in the image are analyzed and classified. Finally, the refined OM with accurate boundaries is used to extract the moving object. Fig. 3 shows an example of moving object extraction. From the segmentation result in Fig. 3(c), we can see the boundaries are very close to the real edge of moving object.

\section{Experiments}

The proposed method is tested on a number of video sequences. In this section, experimental results on Mom-daughter and Table-tennis are presented.

To further evaluate the performance of the proposed method we manually extract the objects in the video sequences as the ground truth or actual objects, and we evaluate the results obtained by the proposed method with the ground truth as follows:

$$
S A=\frac{P_{e}}{P_{a}}
$$

where $S A$ is the spatial accuracy of extracted object. $p_{e}$ is the number of error pixels belonging to the grey regions in Fig. 6(b). $p_{a}$ is the total number of actual object pixels belonging to the grey region in Fig. 6(a).

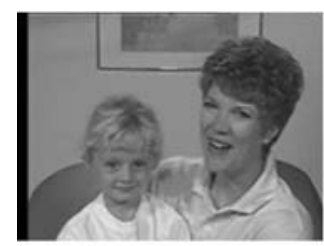

(a)

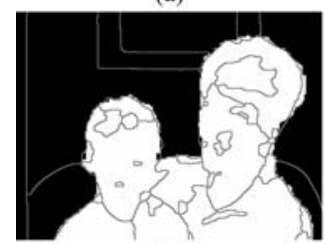

(d)

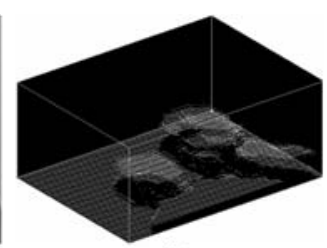

(b)

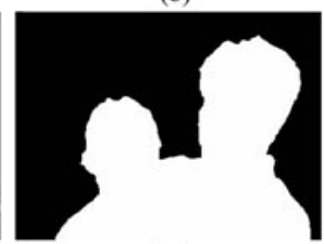

(e)

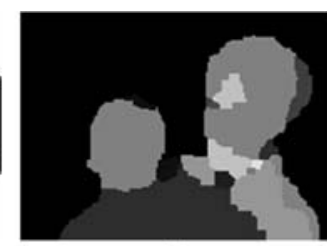

(c)

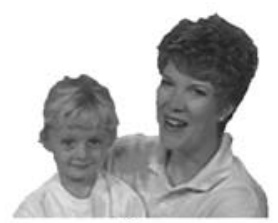

(f)

Fig. 4. Experiment on Mom-daughter: (a) is the original image; (b) gives the extracted motion layers (because the motion of a human body is non-rigid, the motion layers are fluctuant correspondingly, which can also be seen in velocity map (c)); By combining the edge information in (d) with the initial OM, the final results are shown in (e) and (f) 


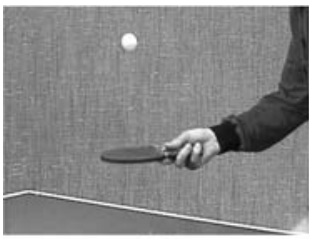

(a)

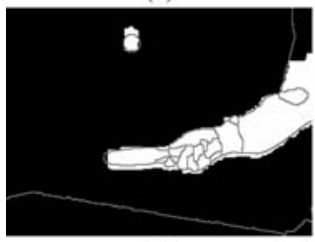

(d)

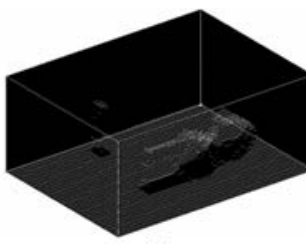

(b)

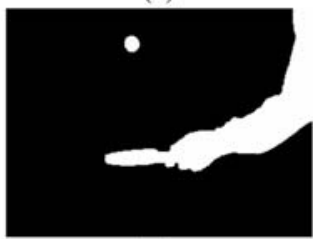

(e)

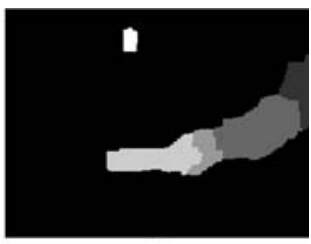

(c)

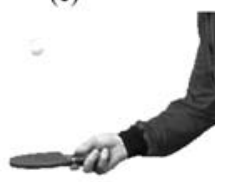

(f)

Fig. 5. Experiment on Table-tennis

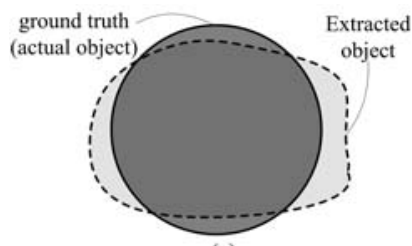

(a)

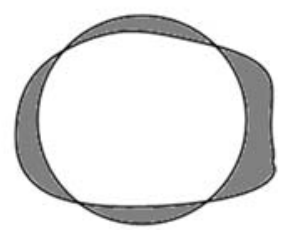

(b)

Fig. 6. Spatial accuracy definition

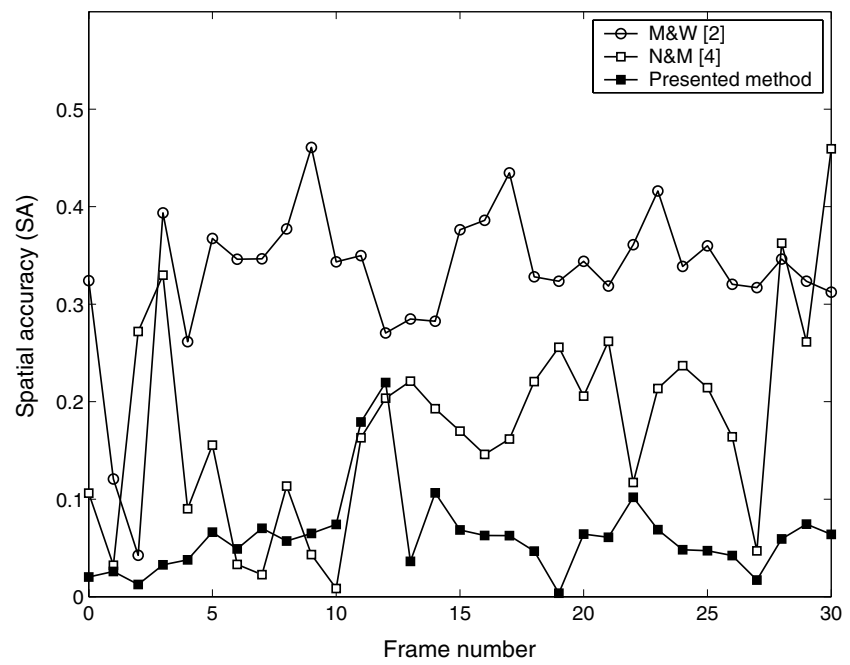

Fig. 7. Evaluation results 
Using such objective performance evaluation criteria, we compare the proposed method with the techniques in [2] and [4]. Fig. 7 shows the evaluation results using Table-tennis sequence. From Fig. 7, we can see that, in many cases, the presented method can produce more accurate segmentation results than the other two. This partly comes from that graph cuts technique can preserve spatial coherency in motion layers, even when there is no manifest texture information. Besides, watershed segmentation together with WCA region merging algorithm also provide satisfying edge information for the extraction of moving objects.

One remaining problem for the proposed method is that only the motion cue between two frames is used to extract motion layer, which is not enough to extract meaningful objects in many cases. We will intend to embed tracking technique into the segmentation system in our future work. Another problem lies in the stage of motion boundary refinement, where the accuracy of object boundaries depends on the edge information too much. If edge map fails to describe the actual boundary information precisely, the extracted object will lose its accuracy.

\section{Conclusion}

This paper presented an approach to automatically segment moving objects from image sequences with accurate boundaries. The contribution of the presented method can be summarized as follows: Motion seed detection, which finds out how many motions are there in the video and where they are, provides a reasonable initial state for Motion layer expansion. Then during the expansion process, energy function and graph cuts technique preserve the spatial coherency of motion layers. Such layers, combined with edge information, produce the segmented moving objects with accurate boundaries. Several experimental results are shown in the paper, which illustrate the promising performance of the proposed method.

\section{Acknowledgements}

This research is sponsored by France Telecom R\&D, and the National Natural Science Foundation of China ( Grant No. 60135020, 60475010 and 60121302 ).

\section{References}

1. Arch, T., Kaup, A.: Statistical model-based change detection in moving video. Signal Processing 31 (1993) 165-180

2. Mech, R., Wollborn, M.: A noise robust method for $2 \mathrm{~d}$ shape estimation of moving objects in video sequences considering a moving camera. Signal Processing 66 (1998) 203-217

3. Meier, T., Ngan, K.: Automatic segmentation of movings for video object plane generation. IEEE Trans. on Circuits \& Systems for Video Technology 8 (2003) 525-538 
4. Nicolescu, M., Medioni, G.: Motion segmentation with accurate boundaries-a tensor voting approach. Proceedings of the IEEE Computer Society Conference on Computer Vision and Pattern Recognition 1 (2003) 382-389

5. Wang, J., Adelson, E.: Layered representation for motion analysis. Proceedings of the IEEE Computer Society Conference on Computer Vision and Pattern Recognition (1993)

6. Darrell, T., Pentland, A.: Cooperative robust estimation using layers of support. IEEE Trans. on Pattern Analysis and Machine Intelligence 17 (1995) 474-487

7. Ayer, S., Sawhney, H.: Layered representation of motion video using robust maximum-likelihood estimation of mixture models and mdl encoding. 5th International Conference on Computer Vision (1995)

8. Jepson, A., Fleet, D., Black, M.: A layered motion representation with occlusion and compact spatial support. 7th European Conference on Computer Vision 1 (2002) 692-706

9. Boykov, Y., Kolmogorov, V.: An experimental comparison of min-cut/max-flow algorithms for energy minimization in vision. IEEE Trans. on Pattern Analysis and Machine Intelligence 26 (2004) 1124-1137

10. Wang, J., Lu, H., Liu, Q.: Moving object segmentation using graph cuts. IEEE Int'l Conf. on Signal Processing 1 (2004) 777-780

11. Vincent, L., Soille, P.: Watersheds in digital spaces: An efficient algorithm based on immersion simulations. IEEE Trans. on Pattern Analysis and Machine Intelligence 13 (1991) 583-598

12. Wang, J., Lu, H., Liu, Q.: A fast region merging algorithm for watershed segmentation. IEEE Int'l Conf. on Signal Processing 1 (2004) 781-784 